\title{
MHUCR: Mutli Hop Uniform Clustering Routing Protocol for Energy Efficient WSN
}

\author{
Salah A. Alabady ${ }^{1 *}$ and Sara Raed ${ }^{2}$ \\ ${ }^{1,2}$ College of Engineering / Computer Engineering Department, \\ University of Mosul / Iraq \\ 1'eng.salah@uomosul.edu.iq, ${ }^{2}$ sararaedl@outlook.com
}

\begin{abstract}
This paper presents an energy efficient routing protocol for wireless sensor network called Mutli Hop Uniform Clustering Routing Protocol (MHUCR). The proposed MHUCR protocol balances the energy consumption of all nodes in the network and prolong the network lifetime. In MHUCR, three types of nodes in the network are assumed. These nodes are defined as, Normal Node (NN), Cluster Head (CH), and Super Node (SN), and each type has a different energy level. The $\mathrm{CH}$ has a fixed number of members, and NN sends a request to join to the $C H$ that has fewer member numbers, close to it, and have a high residual energy as metrics. The SN is assumed to be a near to the Base Station (BS) to overcome the problem of nodes that is near to the BS, which dies faster than distant nodes. MHUCR has the capability to adapt and find another path, in case any change occurs, $\mathrm{CH}$ fails or dies. Four different scenarios using MATLAB are implemented to evaluate and investigate the performances of the proposed protocol. Simulation results show that the proposed MHUCR protocol improvement rate of in terms of nodes still alive was $98 \%$ when compared with LEACH protocol and $91.5 \%$ when compared with LEACH-C protocol and $78.2 \%$ when compared with DECA protocol and $67.5 \%$ when compared with I-LEACH protocol and $41 \%$ when compared with W-LEACH protocol.
\end{abstract}

Keywords: WSN, LEACH, MHUCR, Energy-Efficient Routing, Clustering, W-LEACH

\section{Introduction}

Nowadays, Wireless Sensor Network (WSN) are considered as a one of the most essential technologies due to its used in several applications such as military, health care, industrial and agricultural. WSN consists small, low-cost and low power wireless sensor nodes that operate on battery. These sensor nodes have the ability to measure the specific parameters in the environment such as, temperature, pressure, motion, sound, etc., [1]. The main task of WSN is monitoring the environment. The sensor nodes can communicate among themselves using radio signals. A sensor node consists of four unites sensing, processing, communication and power supply. The processor unit responsible for processing and storing collected data. The sensing unit gathers data from the environment using sensors and converts the data from analog to digital signals using the analog to digital converter (ADC). Communication unit is responsible for providing a communication channel from one node to another node in the network using a transceiver. Lastly, the power-supply unit that is responsible for providing energy to the nodes using a battery that means limited energy resources, and energy must be used in sparingly a way [2]. The communication unit of the sensor node loses the highest power. Therefore, a research in WSNs is mostly focused on designing a protocol that needs as a little power as

Received (January 15, 2018), Review Result (April 17, 2018), Accepted (May 31, 2018)

* Corresponding Author 
possible when communication. Sensor nodes are usually random deployment in inaccessible place and replacement of the energy source are not feasible. Accordingly, an energy efficient routing protocol is a key design issue that needs to be enhanced, in order to improve the life span of the network [3, 4].

In articles, many routing protocols have been proposed and designed for WSNs where energy is an important metric in design issue [2]. General, the routing techniques are classified under three categories based on the underlying network structure: flat, hierarchical, and location-based-routing. In flat-based routing, all nodes have the same function in the network. In location based routing, sensor nodes must be awareness of location to route data across the network by using Global Positioning System (GPS) or any other technique. In hierarchical-based routing, however, nodes will play different roles within the network; the sensor nodes are divided into groups, called clusters. Each cluster has a one node that acts as a leader, called a cluster head $(\mathrm{CH})$, as shown in Figure 1. Clustering in WSN has many advantages, such as: the $\mathrm{CH}$ can discard the redundant data, and this leads to reduce the energy consumption by decreasing the number of packet that needed to send to the BS. Clustering makes routing more simply because only the $\mathrm{CH}$ needed to find the optimal path to the BS. Therefore, requiring a lesser routing information clustering will improve the scalability of the network and additionally, conserve communication bandwidth as the sensor nodes communicate with their $\mathrm{CHs}$ only [5].

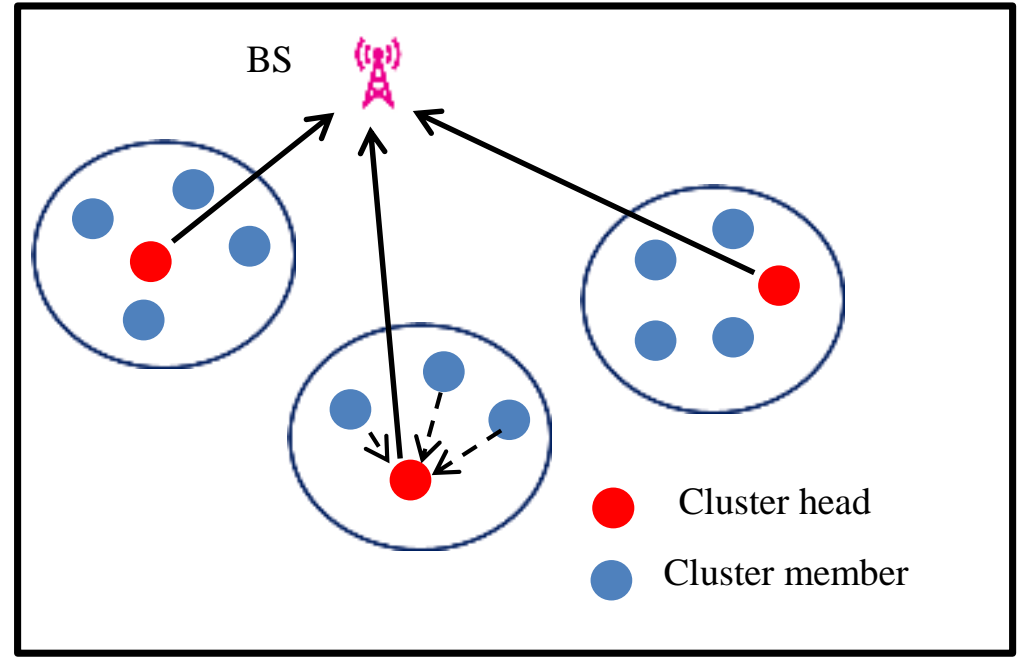

Figure 1. Clustering in WSN

Routing in WSN faces a number of challenges such as: network may have a large number of sensor nodes and deployment in ad-hoc manner; all nodes send data directly or indirectly to the BS. Power source is limited and in some applications, sensor nodes are able to move and change their location.

The objective of this paper is to propose an energy-efficient and reliable routing protocol for WSN using three types of nodes, and each type has a different energy level. SN has a higher energy and placed near to the BS to solve the problem of nodes that are closed to the BS that dies faster than the distant nodes. $\mathrm{CH}$ acts as cluster leader, and has a fixed number of members depended upon the number of $\mathrm{NN}$ in entire a network. $\mathrm{NN}$ acts as a cluster member. To achieve balance in the network, NN connects to closest SN or closest $\mathrm{CH}$ that has more energy and has fewer members.

The rest of the paper is structured as follows. Section 2 summarizes related work. Section 3 presents the system model. Section 4 presents the propose protocol. Section 5 shows the simulation results that show the advantages of the proposed protocol. Section 6 is a conclusion of the paper. 


\section{Related Works}

Sensor nodes are with limited energy resources need the energy efficient routing protocol to increase the network lifetime as much as possible. Routing is the process of choosing a route within a network to send the data to the sink node in an energy awareness method to conserve energy. Many clustering protocols have been proposed for WSN. Low Energy Adaptive Clustering Hierarchy (LEACH) protocol [6] is a one of the first hierarchical routing approaches for sensor networks. LEACH selects a few sensor nodes work as $\mathrm{CHs}$ in each round, in such a way that each node in the network will operate as cluster head and rotates this role to balance the energy conception of nodes. Optimal number of cluster heads is estimated to be $5 \%$ of the total number of nodes. The work of LEACH is separated into two phases, setup phase and steady state phase. In the setup phase, the $\mathrm{CHs}$ are selected. In the steady state phase, the data send to the BS. In setup phase, the node chooses a random number between 0 and 1 . The node becomes a $\mathrm{CH}$ for the current round if the number is less than the following threshold $T(n)$ that is calculated as shown in Eq. (1),

$T(n)=\left\{\begin{array}{cc}\frac{P}{1-P\left(r \bmod \frac{1}{p}\right)} & \text { if } \quad n \in G \\ 0 & \text { otherwise }\end{array}\right\}$

Where, $p$ is a percentage of CHs, $r$ the current round, and $G$ the set of nodes that have not been cluster heads in the last $1 / p$ rounds.

$\mathrm{CH}$ broadcasts to their neighbors in the network the advertisement message for announcing $\mathrm{I}$ a $\mathrm{CH}$ for the current round. If a node receives one advertisement message from one cluster head, then it automatically becomes a member of that cluster. Nodes may receive more than one advertisement message from many $\mathrm{CHs}$ in the network and decide which cluster to be joined based on received the Received Signal Strength Indication (RSSI). Nodes who want to join to the cluster, inform the $\mathrm{CH}$. The $\mathrm{CH}$ will create a cluster and use TDMA schedule for the member node that want to transmit the sensed data. In the steady state phase, the sensor nodes begin sensing and transmitting data to the $\mathrm{CHs}$. The $\mathrm{CHs}$ aggregate data from the nodes in their cluster and sending these data to the BS. At the end of the steady state phase, the network repeats the setup phase again to enter into another round and selecting a new node to act as $\mathrm{CH}$.

The LEACH reduces the power consumption and increase the network lifetime by clustering. Through the steady state phase, only the $\mathrm{CHs}$ are active all the time. A cluster member in a cluster is active only during its allocated time slot and in the setup phase. This leads to minimize the energy consumption of a node. However, LEACH uses singlehop routing where each $\mathrm{CH}$ sends data directly to the sink if sink is far from $\mathrm{CH}$, the $\mathrm{CH}$ needs to consume high energy to send data to sink. Therefore, the LEACH is not appropriate to the networks deployed in the wide areas. In addition, choosing a $\mathrm{CH}$ randomly without relying on the remaining energy of the $\mathrm{CH}$ and the distance between the $\mathrm{CH}$ and the BS is considered as a weakness point. In [7], LEACH-C uses centralized clustering algorithm. The BS collects information from the network nodes to make clustering and routing, after that, the BS send the information of clustering and routing to the sensor nodes in the network. Therefore, at the beginning of each round, the BS must have the location information and the residual energy of every node.

The authors in [8], proposed a new method to Optimize Low Energy Adaptive Clustering Hierarchies (OLEACH) that improve the existing LEACH by selecting cluster according to the residual energy of nodes dynamically. In [9], the author proposed a protocol, Hybrid Energy-Efficient Distributed clustering (HEED) that use residual energy and node density (i.e., number of neighbors) as a metric for cluster selection to achieve power balancing. The HEED clustering increases the network lifetime over LEACH clustering because LEACH randomly selects CHs. In [10], the authors have suggested 
distributed routing algorithms and clustering. The cluster formation is based on the residual energy of $\mathrm{CHs}$, distance between the member sensor nodes to their $\mathrm{CHs}$ and the distance from the $\mathrm{CHs}$ to the $\mathrm{BS}$, but did not take into account that the $\mathrm{CH}$ near the $\mathrm{BS}$ is dying faster than the distant $\mathrm{CH}$.

In [11], the author studies LEACH protocol, and puts forward energy-LEACH and multi-hop LEACH protocols. Energy-LEACH protocol improves the way of choice a $\mathrm{CH}$, makes some nodes have more residual energy as CHs in next round. Multi-hop LEACH protocol improves communication mode from single hop to multi-hop between $\mathrm{CH}$ and sink. Simulation results show that energy-LEACH and multi-hop LEACH protocols have better performance than LEACH protocols. In [12], the authors proposed a new modified enhanced LEACH algorithm that computes the threshold value based on the total energy of nodes in the network for next round, as shown in Eq. (2).

$T(n)=\left\{\frac{P}{1-P\left(\operatorname{rmod} \frac{1}{p}\right)} \times \frac{E o}{\text { Etotal }}\right\} \quad$ if $n \in G$

Where, Eo is the initial energy of node and Etotal is the sum of energy level of all nodes. A comparison between traditional LEACH and proposed LEACH in [12] has been done based on the network lifetime. In [13], the authors proposed routing algorithm ILEACH that selects sensor nodes act as $\mathrm{CH}$ according to higher residual energy, more neighbors, and lower distance from the BS. The WSN lifetime is increased as compared to LEACH and LEACH-C algorithms. In [14], the authors have been proposed routing algorithm that provided a fair chance to nodes that have higher energy to become a cluster head. In [15], the authors proposed a differential evolution based clustering algorithm for WSN. The aim of this proposed [15] is extend network lifetime by avoiding the earlier death of highly loaded CHs. The death of the first $\mathrm{CH}$ can be delayed by choosing a $\mathrm{CH}$ that has less energy, and then reducing the number of members for that $\mathrm{CH}$ by move those members to another $\mathrm{CH}$. The reader can find more details for survey papers that study and analyze different hierarchical routing protocols in [16] and [17]. The protocols in previous related works do not take into consideration the number of member in each cluster. Maybe one of the clusters consist five members and the other nine members, and this leads to an imbalance of energy conception. In addition, the previous related works did not address the problem of nodes close to the base station, which dies faster than distant nodes because the nodes near to the BS always use as intermediate node for transmission data to the BS.

\section{System Models}

\subsection{Energy Model}

There are different methods exist for describing the energy consumption in WSN. At most, the energy expended for amount of $K$ bit data is calculated using Eqs. (3) and (4) [7]:

$E_{T X}(k, d)=E_{\text {elec }} \times k+\varepsilon_{\text {amp }} \times k \times d^{2}$

$E_{R X}(k)=E_{\text {elec }} \times k$

Where, $E_{T X}$ represents the energy required for transmission one packet, $E_{R X}$ represents the energy required for received one packet, $E_{\text {elec }}$ is the energy needed for modulating or demodulating one bit. $\varepsilon_{\text {amp }}$ is the consumed energy of amplifier, $k$ represents the packet size and $d$ represents the distance between a sender and a receiver. 


\subsection{Network Model}

In the MHUCR, three types of node deploy randomly over a square area is assumed. Each type has different level of energy. The type of nodes as following: Normal Node (NN) has less energy because it only sends data to the nearest $\mathrm{CH}$ or nearest $\mathrm{SN}$. $\mathrm{CH}$ has energy more than $\mathrm{NN}$ because it receives data from cluster member and sends the received data to the nearest $\mathrm{CH}$ or nearest $\mathrm{SN}$. Super Node $(\mathrm{SN})$ has high energy and solar panel for renewable energy. SN receives data from $\mathrm{CH}$ and nearest $\mathrm{NN}$ and send the received data to the nearest $\mathrm{SN}$ or to the $\mathrm{BS}$. A node can compute the distance to another node based on the RSSI. The BS (i.e., sink node) located in the center of sensor filed, and the $\mathrm{CH}$ and $\mathrm{SN}$ use multi-hop routing to send data to that BS. Similar to LEACH, gathering the data is divided into rounds. In each round, all sensor nodes sense environment and send data to $\mathrm{CH}$ or SN. Between two rounds, nodes turn off their radios to save energy.

\section{Propose MHUCR Psrotocol}

Limit energy of WSN motivates to propose MHUCR in order to increase the network lifetime as much as possible. Several assumptions are made in the proposed model; these assumptions are described as follows:

- All sensor nodes include $\mathrm{NN}, \mathrm{CH}$ and $\mathrm{SN}$, in addition to the BS are stationary after deployment and location unaware.

- All nodes have similar capabilities (processing/communication), but are different in terms of energies in case of heterogeneity.

- Nodes distribution is random in sensor filed.

- The number of BS depends on the size of WSN.

- $\mathrm{CH}$ nodes broadcast advertising message within the communication range of a sensor node. The advertising message consists of cluster head identifier ( $\mathrm{CH}$-ID), residual energy, distance to the BS and the number of members they have.

- All normal nodes have received at least two advertising message.

- NNs send request message to join to $\mathrm{CH}$ that has maximum residual energy, closest to her, less distance to the BS and has lower number of members.

- If $\mathrm{CH}$ has max number of members, $\mathrm{NN}$ sends data to the second closer $\mathrm{CH}$ or to $\mathrm{SN}$.

- If $\mathrm{CH}$ dies, the members of this $\mathrm{CH}$ send data to second nearer $\mathrm{CH}$ or to $\mathrm{SN}$.

Figure 2 shows the pseudo-code of MHUCR.

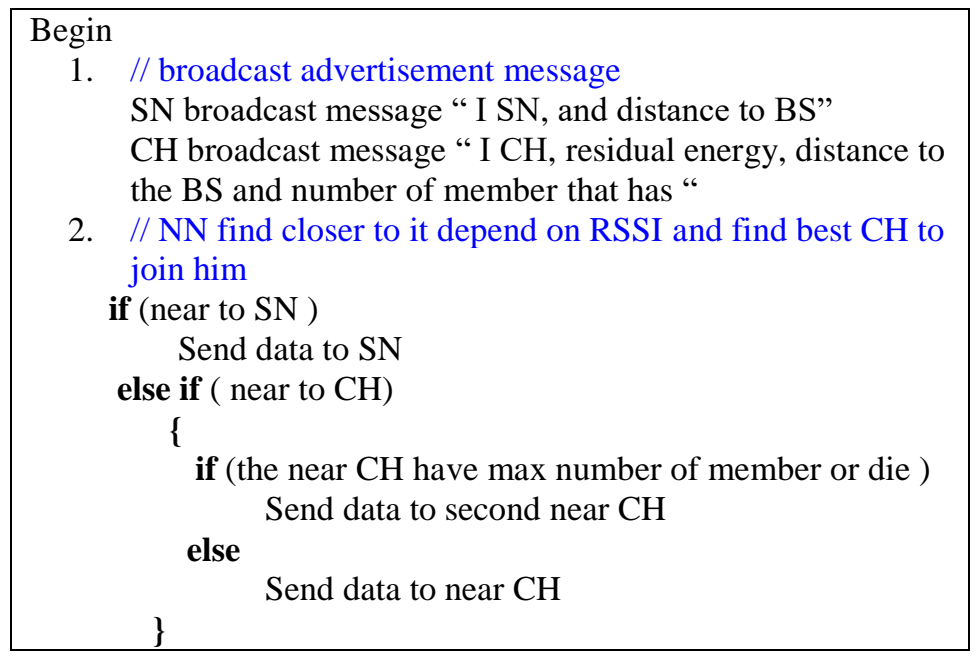




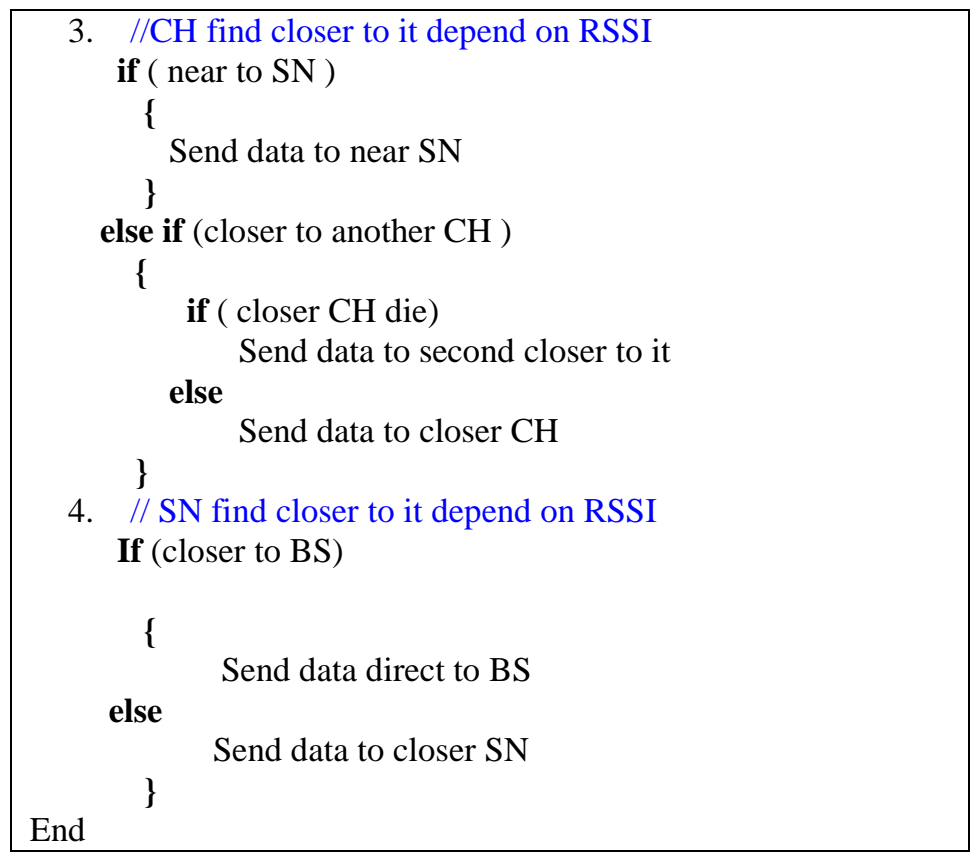

Figure 2. MHUCR Pseudo-Code of

\section{Experiment Results}

The MHUCR protocol is implemented using MATLAB software. To investigate the performance of the MHUCR, four scenarios have been applied. The first scenario presents the performance of MHUCR. In the second, third and fourth scenarios a comparison with other protocols, such as, W-LEACH [14], LEACH [6], DECA [15], I-LEACH [13], and LEACH-C [7] are implemented. In each scenario, the experiments are performed with different values of number of nodes, initial energy of nodes, packet size, max number of member in each $\mathrm{CH}$, and the area of sensor filed. In all scenarios, a node is dead if its energy level reaches to 0 joules.

Table 1. Simulation Parameters of the Four Scenarios

\begin{tabular}{|c|c|c|c|c|}
\hline Parameter & $\begin{array}{c}\text { First } \\
\text { Scenario } \\
\end{array}$ & $\begin{array}{c}\text { Second } \\
\text { Scenario }\end{array}$ & $\begin{array}{c}\text { Third } \\
\text { Scenario }\end{array}$ & $\begin{array}{c}\text { Fourth } \\
\text { scenario } \\
\end{array}$ \\
\hline Normal Nodes(NN) & 80 & 84 & 100 & 180 \\
\hline $\mathrm{CH}$ nodes $(\mathrm{CH})$ & 20 & 16 & 15 & 20 \\
\hline $\begin{array}{l}\text { Number of node } \\
(\mathrm{NN}+\mathrm{CH})\end{array}$ & 100 & 100 & 115 & 200 \\
\hline Super Nodes $(\mathrm{SN})$ & 8 & 8 & 8 & 8 \\
\hline Max clusters member & 4 & 5 & 6 & 9 \\
\hline Sensor filed & $200 \times 200 \mathrm{~m}$ & $50 \times 50 \mathrm{~m}$ & $200 \times 200 \mathrm{~m}$ & $200 \times 200 \mathrm{~m}$ \\
\hline Initial energy of NN & 0.9 joule & 0.5 joule & 2 joule & 0.5 joule \\
\hline Initial energy of $\mathrm{CH}$ & 4 joule & 3 joule & 10 joule & 5 joule \\
\hline Initial energy of SN & 10 joule & 6 joule & 15 joule & 15 joule \\
\hline$E_{\text {elec }}$ & $100 \mathrm{~nJ} / \mathrm{bit}$ & $50 \mathrm{nj} / \mathrm{bit}$ & $50 \mathrm{nj} / \mathrm{bit}$ & $50 \mathrm{nj} / \mathrm{bit}$ \\
\hline$\varepsilon_{a m p}$ & $100 \mathrm{pJ} / \mathrm{bit}$ & $100 \mathrm{pj} / \mathrm{bit}$ & $0.0013 \mathrm{pj} / \mathrm{bit}$ & $\begin{array}{c}0.0013 \\
\mathrm{pj} / \mathrm{bit}\end{array}$ \\
\hline$\varepsilon_{f s}$ & $0.013 \mathrm{pJ} / \mathrm{bit}$ & $10 \mathrm{pj} / \mathrm{bit}$ & 10pj/bit & $10 \mathrm{pj} / \mathrm{bit}$ \\
\hline Packet size & 1600 bit & 2000 bit & 4000 bit & 4000 bit \\
\hline
\end{tabular}




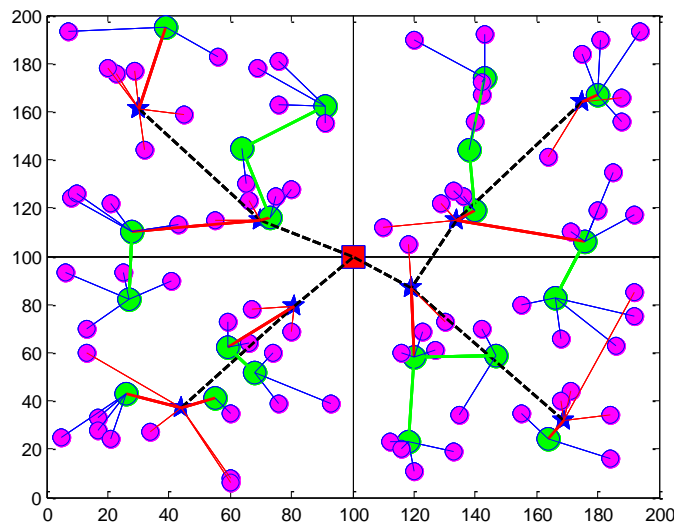

(A) First Scenario

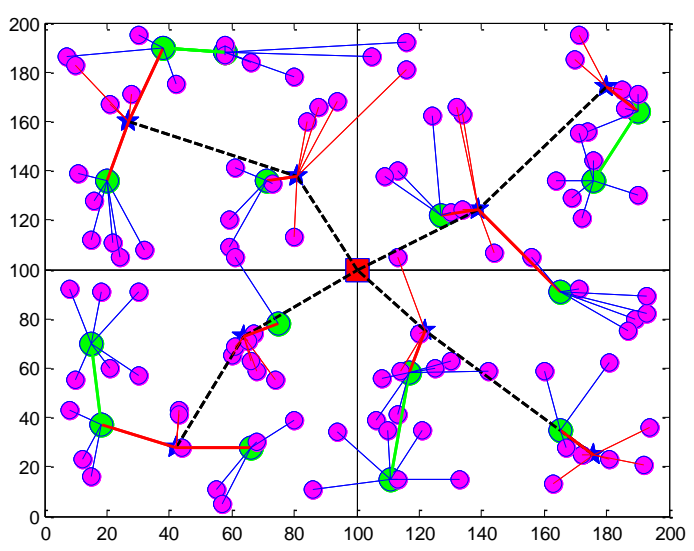

(C) Third Scenario

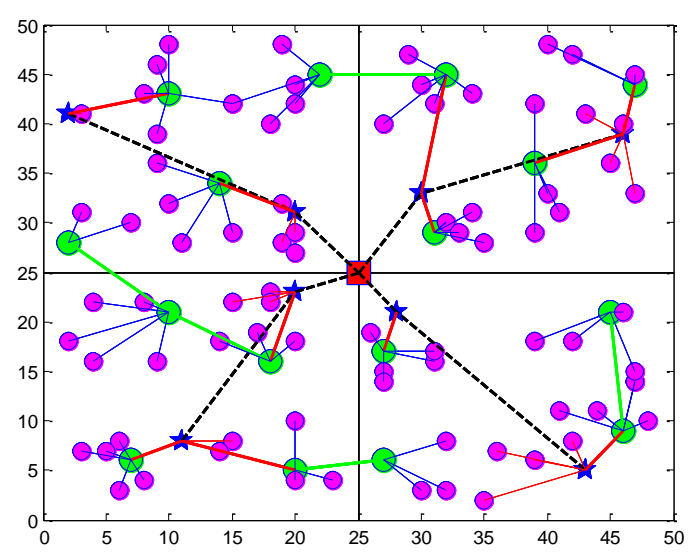

(B) Second Scenario

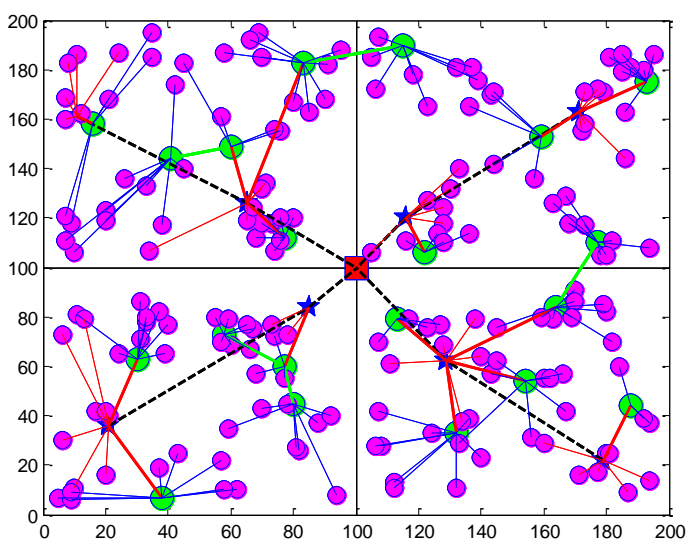

(D) Fourth Scenario

\section{Figure 3. Network Topology of Four Scenarios where: \\ Base Station \\ $\star \quad$ Super Node \\ Cluster Head \\ Normal Node}

\subsection{First Scenario}

The parameters values that are used in the first scenario are summarized in Table 1 . The wireless sensor nodes are distributed randomly within an area of $200 \times 200 \mathrm{~m}^{2}$. The position of the BS is chosen at the center of that area with coordinates $(100,100)$. Figure 3(A) captures a random snapshot of nodes distribution from a randomly chosen simulation Figure 4(A) shows the number of nodes that still work (i.e., live) versus the number of rounds for the proposed MHUCR protocol. The figure demonstrated that the number of nodes that still live would be decreased when the number of rounds increases. For the 1400 round, the number of nodes that still live is 95 , while it is 71 and 43 for 3000 and 4000 rounds, respectively. As shown in Figure 4(A), there is about $50 \%$ of nodes number still live at the 4000 round.

Figure 4(B) shows the residual energy versus the number of rounds when the area of the sensor field is changed to study the effect of the distance on the amount of energy consumed, the remaining energy decreases when increased the area of the sensor field because the greater the transmission distance, the greater the energy consumed in each 
round, where the energy is consumed by the sensitivity and the sending and receiving of data.

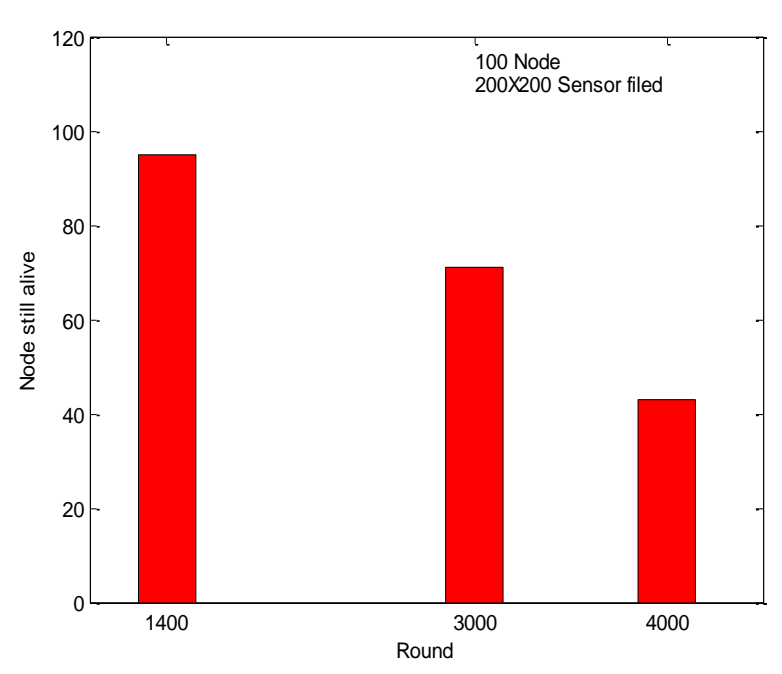

(A)

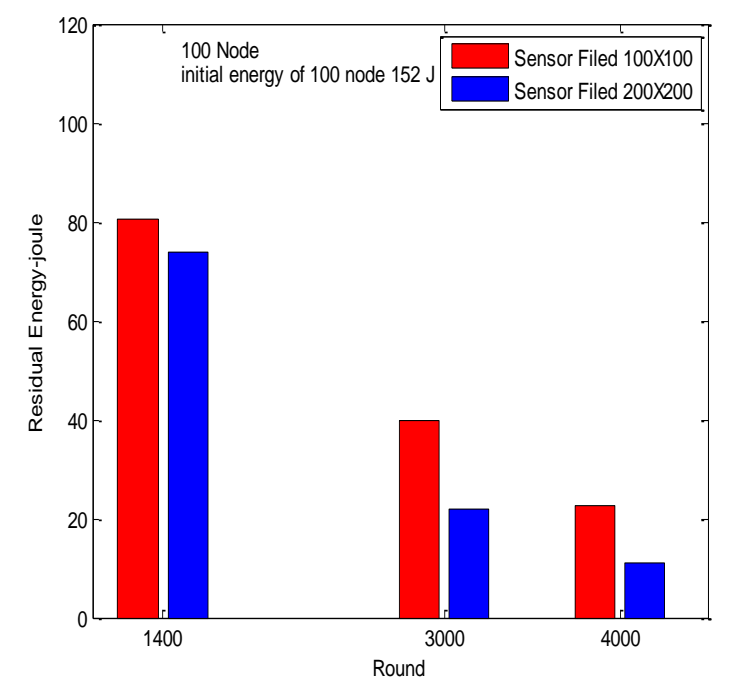

(B)

Node Still Alive versus Number of Round

Residual Energy versus Number of Round

Figure 4 (A, B). Simulation Results of the First Scenario

Figure 5 shows the simulation results that obtained from the first scenario for the MHUCR that compared with the improved W-LEACH [14] in relation of node that still alive versus number of round. Figure 5 shows that, when the number of rounds is 1400 , the number of node that is still alive is 98 for improved W-LEACH, and it is 89 for MHUCR. However, at 3000 round, the number of nodes that is still alive are 71 for MHUCR, and 30 for improve W-LEACH. The results show that the improvement rate of the MHUCR protocol in terms of nodes still alive was $41 \%$ when compared with $\mathrm{W}$ LEACH protocol.

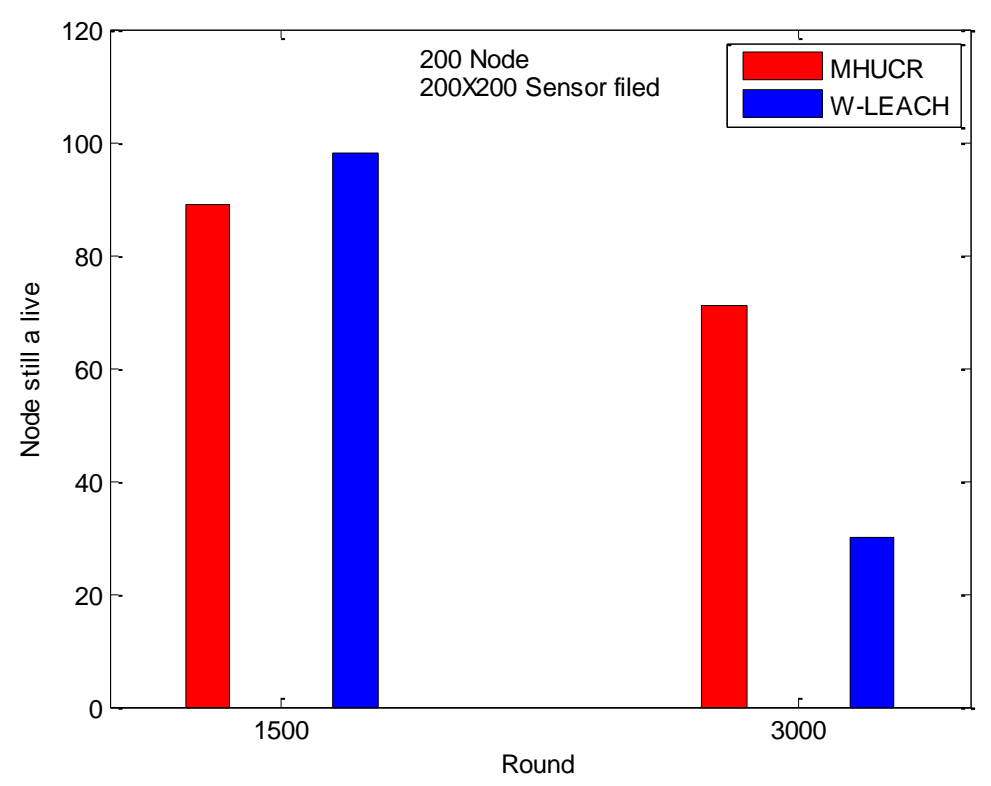

Figure 5. Simulation Results Comparison between MHUCR and Improved W-LEACH Protocol 


\subsection{Second Scenario}

The values of the system parameters used in the second scenario are summarized in Table 1. The sensing field is $50 \times 50 \mathrm{~m}^{2}$ and the position of the BS is taken at the center of that area. In this scenario, a comparison between MHUCR and LEACH [6] is made. Figure. 3(B) shows the capture of a random snapshot of nodes that randomly distributed. The differences between the first and second scenarios are that, in the second scenario, the area of sensor filed is reduced to examine the effect of distance on sensor nodes and change the number of $\mathrm{CH}$, max number of member for each $\mathrm{CH}$, initial energy of all nodes, and the packet size.

Figure 6 shows the comparison results in relation of node still alive and round. Note that the number of nodes that still alive after run 1400 round in LEACH is equal to zero, while in MHUCR, there are 98 nodes still alive (from 100 nodes). The advantage of this result since the MHUCR take into account that $\mathrm{NN}$ send request join to $\mathrm{CH}$ that have less member and closer to it and have higher residual energy. Thus, this leads to a balance in energy dissipation in whole network. Figure 6 is illustrations the number of nodes that still alive for MHUCR in case 3000 and 4000 rounds is 90 and 88, respectively. To investigate the performance of the MHUCR, and to check at which round all nodes will be dead under the parameters that are shown in Table 1, the simulation program running at different number of rounds. The simulation results show that at 5000 round all nodes will be dead while in the LEACH, all nodes are already dead at 1400 round. In conclusion, the MHUCR increases the network lifetime. The results show that the improvement rate of the MHUCR protocol in terms of nodes still alive was $98 \%$ when compared with LEACH protocol

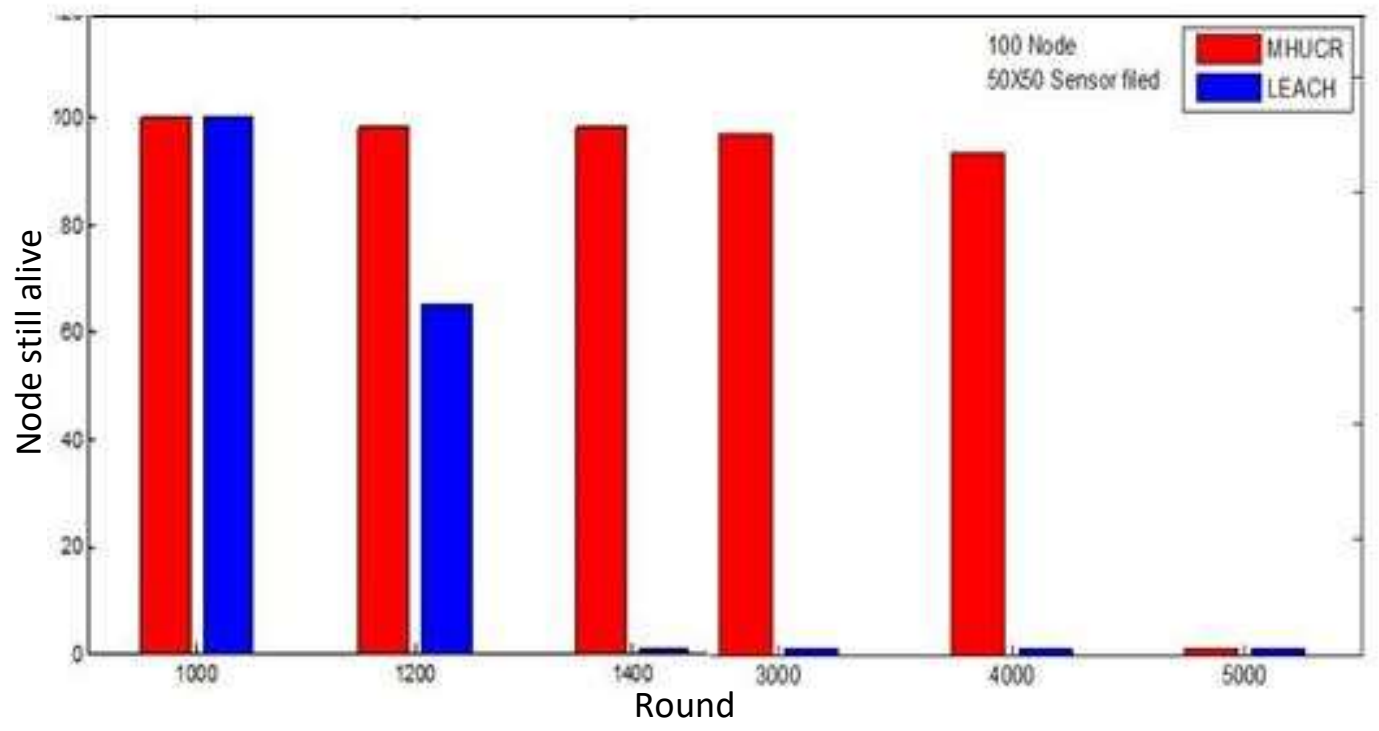

Figure 6. Simulation Results Comparison between MHUCR and LEACH Protocols

\subsection{Third Scenario}

Table 1 shows the values of the system parameters that are used in the third scenario. In this scenario, the sensing field is $200 \times 200 \mathrm{~m}^{2}$ and the position of the BS is at the center of that area. A comparison between MHUCR and DECA [15] is presented. Figure 3(C) shows the capture of a random snapshot of nodes that randomly distributed for third scenario. In this scenario, the number of sensor nodes, initial energy of all nodes, max 
number of member for each $\mathrm{CH}$, and packet size are increased to study the effect of change these parameter on number of node still a live and network lifetime.

Figure 7 shows the comparison results between the MHUCR and DECA in relation of node still alive and round. Figure 7 shows that the number of nodes that still alive after run 1600 and 1800 round in DECA is equal to 75 and 25, respectively while in MHUCR, all nodes still alive (115 nodes). The advantage of this result since the MHUCR take into account that $\mathrm{NN}$ send request join to $\mathrm{CH}$ that have less member and closer to it and have higher residual energy. In addition, SN provides its effectiveness and ability to increase the lifetime of the network. Thus, this leads to a balance in energy dissipation in whole network. The results show that the improvement rate of the MHUCR protocol in terms of nodes still alive was $78.2 \%$ when compared with DECA protocol.

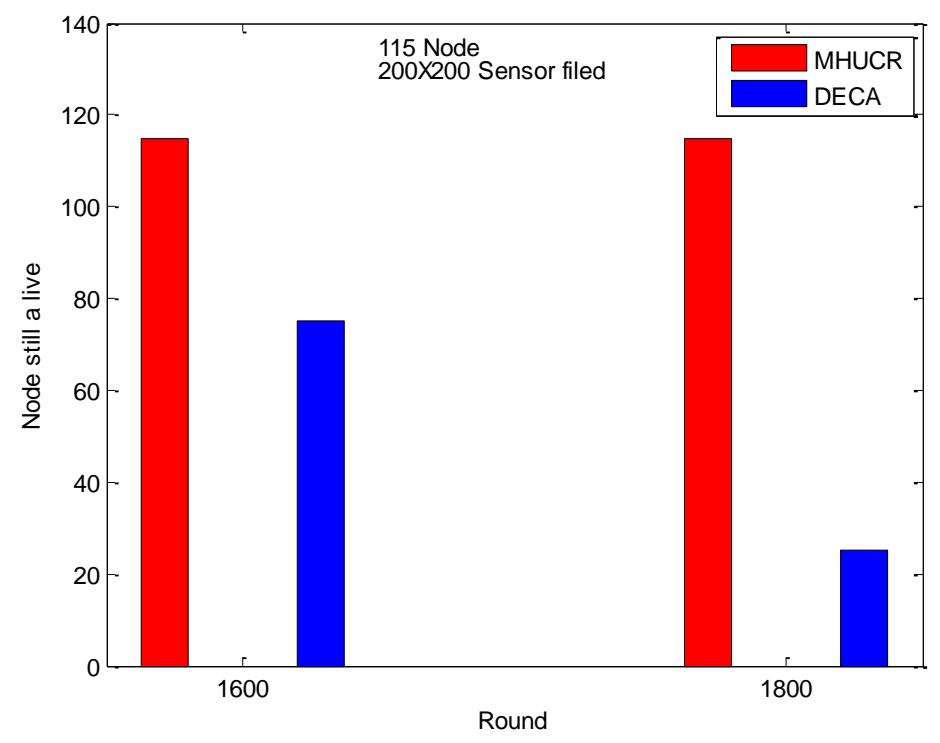

Figure 7. Simulation Results Comparison between MHUCR and DECA Protocols

\subsection{Fourth Scenario}

The values of the system parameters used in the fourth scenario are summarized in Table 1. The sensing field is $200 \times 200 \mathrm{~m}^{2}$ and the position of the BS is taken at the center of that area. In this scenario, a comparison between MHUCR with I-LEACH [13] and compare MHUCR with LEACH-C [7] is presented. Figure 3(D) shows the capture of a random snapshot of nodes that randomly distributed. In this scenario, we also increase the number of sensor node and change initial energy of all nodes, max number of member for each $\mathrm{CH}$ to study the effect of change these parameters on number of node still a live and network lifetime.

Figure 8 shows the comparison results in relation of node still alive and round. One can note, from Figure 8, that the number of nodes that still alive after run 1500 round in ILEACH and LEACH-C is equal to 60 and 20 respectively, while in MHUCR there are 187 nodes still alive (from 200 nodes). In case the number of rounds is increased, all nodes of LEACH-C will be died, and 48 nodes remain with the I-LEACH, while 183 nodes remain alive with MHUCR and only four nodes died. The results show that the improvement rate of the MHUCR protocol in terms of nodes still alive is $91.5 \%$ when compared with LEACH-C protocol and $67.5 \%$ when compared with I-LEACH. 


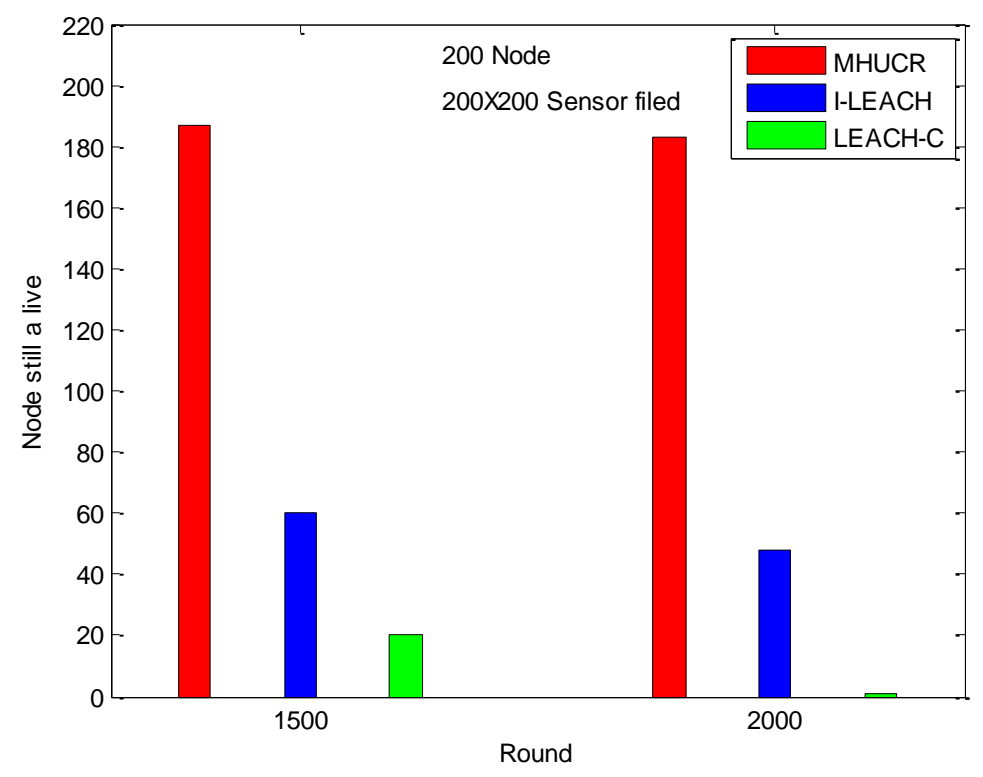

Figure 8. Simulation Results Comparison between MHUCR and I-LEACH, LEACH-C Protocols

\section{Conclusion}

Wireless Sensor Networks (WSN) is used in different applications in which the lifetime is the most important factor. Consequently, need to reduce the energy consumption in WSN to save limited energy and increase the lifetime of the network as much as possible. In this paper, we propose a Mutli Hop Uniform Clustering Routing (MHUCR) protocol to balance energy consumption in a whole network and use a limited energy in efficient manner to prolong network lifetime. The MHUCR has the capability to adapt and find another path, in case any change occurs, $\mathrm{CH}$ fails or dies. The performance of our proposed code, MHUCR, is evaluated using different scenarios with different parameters values. Comparisons are made between the MHUCR protocol with others protocols. Results indicate that the performance of the MHUCR is better than DECA, ILEACH, LEACH-C, and Improved W-LEACH protocols in terms of nodes still alive and residual energy. MHUCR routing protocol improves the lifetime of the network and overcomes the problem of nodes that near to the BS that die quickly. In addition, simulation results show that the MHUCR protocol balances the energy consumption of all nodes and increases the network lifetime.

\section{References}

[1] K. M. Modieginyane, B. B. Letswamotse, R. Malekian, and A. M. Abu-Mahfouz, "Software defined wireless sensor networks application opportunities for efficient network management: A survey", Computers and Electrical Engineering, vol. 66, (2018), pp. 274-287.

[2] N.A. Pantazis, S.A. Nikolidakis, and D.D. Vergados, "Energy-Efficient Routing Protocols in Wireless Sensor Networks: A Survey," IEEE Communications surveys \& tutorials, vol. 15, no. 2, (2013), pp. 551-591.

[3] Matin, M.A. and Islam, M.M., "Overview of Wireless Sensor Network," In Wireless Sensor NetworksTechnology and Protocols, InTech, (2012).

[4] R. Hawi, "Wireless Sensor Networks-Sensor Node Architecture and Design Challenges", International Journal of Advanced Research in Computer Science, vol. 5, no. 1, (2014), pp.47-53.

[5] J.N. Al-Karaki, and A.E. Kamal, "Routing Techniques in Wireless Sensor Networks: A Survey", IEEE wireless communications, vol.11, no.6, (2004), pp. 6-28.

[6] W.R. Heinzelman, J. Kulik, and H. Balakrishnan, "Adaptive Protocols for Information Dissemination in Wireless Sensor Networks", In Proceedings of the 5th annual ACM/IEEE international conference on Mobile computing and networking, Washington, USA, (1999), pp. 174-185. 
[7] W. B. Heinzelman, A. P. Chandrakasan, H. Balakrishnan, "An Application-Specific Protocol Architecture for Wireless Sensor Networks", IEEE Trans. on Wireless Communications, vol. 1, no. 4, (2002), pp. 660-670.

[8] S.E. Khediri, N. Nasri, A. Wei and A. Kachouri, "A New Approach for Clustering in Wireless Sensors Networks Based on LEACH”, Procedia Computer Science, vol. 32, (2014), pp.1180-1185.

[9] O. Younis and S. Fahmy, "HEED: A Hybrid, Energy-Efficient, Distributed Clustering Approach for Ad Hoc Sensor Networks", IEEE Transactions on Mobile Computing, vol. 3, no. 4, (2004), pp.366-379.

[10] M. Azharuddin, P. Kuila, and P.K Jana, "Energy Efficient Fault Tolerant Clustering and Routing Algorithms for Wireless Sensor Networks," Computers \& Electrical Engineering, vol. 41, (2015), pp.177-190.

[11] F. Xiangning and S. Yulin, "Improvement on LEACH Protocol of Wireless Sensor Network", in Proceeding SENSORCOMM International Conference on Sensor Technologies and Applications, Valencia, Spain, (2007), pp. 260-264.

[12] S. Anam and O.P. Yadav, "Performance Enhancement of Leach Protocol in Wireless Sensor Network in Terms of Network Life Time," International Journal for Technological Research in Engineering, vol. 4, no. 7, (2017), pp.1060-1063.

[13] Z. Beiranvand, A. Patooghy and M. Fazeli, "I-LEACH: An Efficient Routing Algorithm to Improve Performance and to Reduce Energy Consumption in Wireless Sensor Networks", In 5th IEEE Conference on Information and Knowledge Technology (IKT), Shiraz, Iran, (2013), pp. 13-18.

[14] Rad, F., Moghtaderinasab, Z. and Parvin, H., "An Improved W-LEACH Routing Protocol in Wireless Sensor Network," Journal of Advances in Computer Research, vol. 8, no. 2, (2017), pp. 39-51.

[15] P. Kuila and P.K. Jana "A Novel Differential Evolution Based Clustering Algorithm for Wireless Sensor Networks," Applied Soft Computing, vol. 25, (2014), pp. 414-425.

[16] D.R. Prasad, P.V. Naganjaneyulu and K.S. Prasad, "Modified LEACH Protocols in Wireless Sensor Networks-A Review", In Proceedings of 2nd International Conference on Micro-Electronics, Electromagnetics and Telecommunications, (2017), pp. 681-688.

[17] G. Rama Subba Reddy and S. Balaji, "A Review on Different Types of LEACH Protocol for Wireless Sensor Networks", In International Journal of Scientific Research in Computer Science, Engineering and Information Technology, vol. 2, no. 4, (2017), pp. 840-844.

\section{Authors}

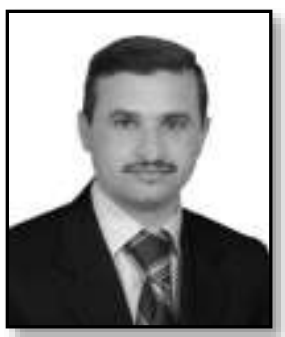

Salah Abdulghani Alabady received the Ph.D. degree in computer engineering/wireless networks from the School of Electrical and Electronic Engineering, Universiti Sains Malaysia, Pulau Pinang, Malaysia, in 2014. From 1999 to 2010, he was a Lecturer with the Computer Engineering Department, University of Mosul, Iraq, where he is currently a Senior Lecturer. From 2011 to 2014, he was a Research Assistant in wireless networks with the School of Electrical and Electronic Engineering, Universiti Sains Malaysia. His research interests include wireless channel coding, joint channel-network coding, error correction codes, and cross layer of wireless sensor networks.

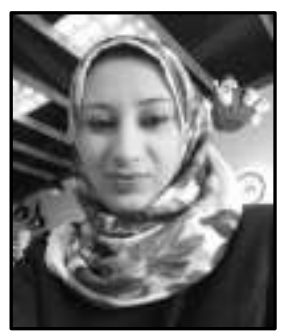

Sara Raed received the B.Sc. degree in Computer Engineering from Mosul University, Mosul, Iraq, in 2013. She is currently working toward the M.Sc. degree in Computer Engineering at the College of Engineering in Mosul University. She is interested in doing research in wireless sensor networks, energy-efficient communication protocols for WSNs, cross-layer design for WSNs and Internet of Thing. 